\title{
An Efficient Design of RPL Objective Function for Routing in Internet of Things using Fuzzy Logic
}

\author{
Adeeb Saaidah $^{1}$, Omar Almomani $^{2}$ \\ Computer Network and Information Systems Department \\ Faculty of Information Technology, The World Islamic \\ Sciences \& Education University Amman, Jordan
}

\author{
Laila Al-Qaisi ${ }^{3}$ \\ Computer Science Department, Faculty of Information \\ Technology, The World Islamic Sciences \& Education \\ University Amman, Jordan
}

\author{
Mohammed Kamel MADI ${ }^{4}$ \\ Faculty of Computing and Information Technology, Sohar University, Sohar, Sultanate of Oman
}

\begin{abstract}
The nature of the Low power and lossy networks (LLNs) requires having efficient protocols capable of handling the resource constraints. LLNs consist of networks that connect different type of devices which has constraints resources such as energy, memory and battery life. Using the standard routing protocols such as Open Shortest Path First (OSPF) is inefficient for LLNs due to the constraints that LLNs need. So, IPv6 Routing Protocol for Low-Power and Lossy Networks (RPL) was developed to accommodate these constraints. RPL is a distance vector protocol that used the object functions (OF) to define the best tree path. However, choosing a single metric for the $O F$ found to be unable to accommodate applications requirements. In this paper, an enhanced (OF) is proposed namely; OFRRT-FUZZY relying on several metrics combined using Fuzzy Logic. In order to overcome the limitations of using a single metric, the proposed OFRRT-FUZZY considers node and link metrics. Namely, Received Signal Strength Indicator (RSSI), Remaining Energy (RE) and Throughput (TH). The proposed OFRRT-FUZZY is implemented under Cooja simulator and then results were compared with OFO, MHROF in order to find which OF provides more satisfactory results. And simulation results show that OFRRT-FUZZY outperformed OF0 and MHROF.
\end{abstract}

\section{Keywords-RPL; Objective Function; IOT; fuzzy logic; LLNs}

\section{INTRODUCTION}

The revolution of networks is changing rapidly, starting from Internet to smart phone until Internet of Things (IoT) glare. This revolution is driven by the capabilities of (IoT) to provide benefits for any system either that user or business [1]. This benefits to ease human life through allowing devices to make tasks instead of human [2]. IoT represents the future of computing and communications in several fields from wireless sensors to nanotechnology. IoT comes from the two words "Internet" and "Things" which means that IoT networks connect different things which forms a heterogonous system such as sensors, monitors and smart devices [3][1]. These heterogonous devices can be able to collects data, make communication, computation and ultimate decision making [4]. There are many definitions for IoT, the best one is explained by [5]: "An open and comprehensive network of intelligent objects that have the capacity to auto-organize, share information, data and resources, reacting and acting in face of situations and changes in the environment" [1]. As a result, the rapid increase of number of things which are connected to the network while some of them have limited resources, needs more attention in order to handle all these constraints for LLNs.

One of the critical issues in IoT is the constraints for those devices that connected in LLNs [6][7]. These constraints such as battery power which means it has limited life time, short transmission range, in addition to noisy environment arrangement in LLNs, needs particular protocol to handle the increasing of number of IoT devices and the constraints in order to provide a robust routing of data and efficiency [5][7][8][9]. As a result, the Internet Engineering Task Force (IETF) developed a routing standardized protocol for 6LoWPAN networks which is called RPL [10]. RPL overcomes and handle all the constraint requirements that need for LLNs. Furthermore, It can adapt the variation of link and node metrics over time. Considering that LLNs nodes can have one or several parent which forms a route to the root.

RPL is the main candidate for acting as the standard routing protocol for IPv6 based LLNs, like wireless sensor networks [11]. It has been proposed as a tree routing protocol by the roll working group. It is also an extensible and flexible single path protocol. Nevertheless, it only saves an optimal routing at a specific time. It chooses a parent node of the best parent nodes [12]. It uses an OF to find the best path. Moreover, the chosen metrics to define the best path aren't defined by the working group. RPL uses a set of constraints and metrics through specific OF for building a Destination Oriented Directed Acyclic Graph (DODAG). The OFs choose the best parent of nodes for building and optimizing the route. However, the standard OF suffer from several limitations which are attributed to the single metric usage. In order to solve the limitations of employing a single metric, a new OF based on combined metrics using Fuzzy Logic is proposed. This OF, considers the node and the link metrics, RSSI, RE and TH.

The second part of this study aims at discussing RPL routing protocol. The third part aims at reviewing the relevant works, whereas the fourth part aims at discussing the metrics. The fifth part sheds a light on the proposed OFRRT_FUZZY algorithm. The sixth part presents the outcomes of the 
developed simulation. The seventh part presents the conclusion.

\section{RPL ROUTING PROTOCOL}

RPL was designed by ROLL working group from IETF to cover the limited resources that attached to the connected devices in LLNs called RPL [13][14]. These limited resources, such as battery life, memory and connectivity to the Internet. The existing routing protocol such as OSPF unable to handle these limitations for these reasons RPL considered as a standard routing protocol for LLNs. RPL is an IPV6 distance vector routing protocol [15] that use the mechanism of Directed Acyclic Graphs (DAGs) [16][17] to apply a tree structure between nodes. Hence each node could be having more than one parent each one could be a next hop in a path to reach a sink. The nodes organized as (DODAGs) [18] where the best parent are chosen based on the metrics. The information exchange between nodes by using the following ICMPv6 control messages [10][19].

- DODAG Information Object (DIO): It's employed for storing the information needed for creating the upward routes of DODAGs such as current Rank, DODAG ID, etc.

- Destination Advertisement Object (DAO): It's employed for sending destination information (path information) upwards to the root along the DODAG.

- DODAG Information Solicitation (DIS): It's employed for enabling the node to solicit a DODAG information object (DIO) from a reachable neighbor.

- Destination Advertisement Object Acknowledgement (DAO-ACK): Unicast message sent by recipient node to acknowledge DAO message.

Fig. 1 show the node working once receives DIO messages and to select the best parents based on calculated rank.

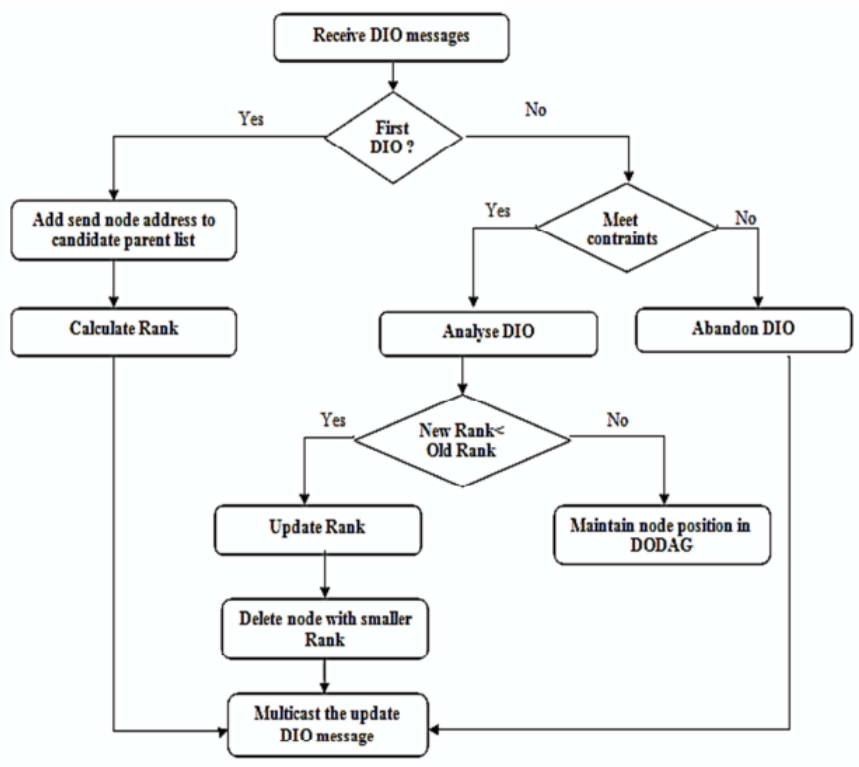

Fig. 1. Different Operations Performed after Receiving a DIO and the Process of Rank Calculation in RPL.

\section{RELATED WORK}

OF is considered as vital part of RPL. It determines major decisions such as parent selection and forwarding path. OF is a hot topic researchers in the field got interested to investigate in order to improve RPL performance. Moreover, fuzzy logic was chosen to be a major addition to OF in RPL.

In [20] Gaddour et al., designed a Fuzzy Logic OF which combined a set of metrics namely; Hop Count, End-to-End Delay, Node Energy and Link Quality. These were used as fuzzy parameters to configure a routing decision. The OF designed, took the application requirements in to consideration for the purpose of finding best path to destination. The evaluation was conducted using large-scale test bed in Contiki OS and Cooja Simulations showed that OF had registered a great improvement in the RPL-based LLNs compared to other used OF.

While [21] Kamgueu et al., proposed a fuzzy inference system for getting better performance than other used systems. They saw that routing systems usually tend to rely on network lifetime without referring to other network performance metrics. As a result, they combined expected transmission delay, count, and node's remaining power. Their Implementation was conducted on Contiki OS and simulations were performed on Cooja. Results showed the combined metrics OF registered significant improvements among one metric OF especially the ETX scenario.

In [22] Kamgueu et al., developed a new RPL OF which uses a fuzzy inference system for combining several metrics (i.e. the expected transmission delay, count, and node's remaining power). The assessment was conducted through using a real sensor network which was deployed in an indoor environment. The results showed that combined fuzzy OF performed better than ETX based routing on energy efficiency, packet loss ratio, routing stability, and end-to-end delay.

In [23] Lamaazi and Benamar, proposed a combined metrics based on fuzzy logic OF. It was designed with node and link metrics (i.e. hop count and energy consumption \& expected transmission count). Their results showed that combined OF-Fuzzy outperformed OF based ETX and OF based energy consumption in terms of overhead and Packet Delivery Ratio (PDR). It was found that OF-Fuzzy participated in equalizing nodes' energy consumption through the network.

Another research conducted by [24] Aljarrah proposed a multi fuzzy logic model for OF for RPL (Ml-FL) consisting of three vital metrics: node-oriented metrics, channel-oriented metrics and link-oriented metrics for uncasing. The Ml-FL chose the best parent for uncast through nine individual metrics. Three other parameters were used to define each of the nine metrics to ensure effective parent node selection. To overcome fuzzy logic complexity, multiple fuzzy logic blocks were processed in parallel. Moreover, an enhanced- BMRF algorithm is proposed with the minimum of delay and duplicate packets. IEEE 802.15.4 standard was applied over OMNeT++ simulator for assessing the effectiveness of the proposed RPL. The outcomes reached through the proposed RPL are promising in terms of energy, end to end delay, hop count, packet delivery ratio and packet loss rate. 
In [25] Fabian et al., stated that amount of data represented by IoT is rapidly growing. While wireless sensors discompose a great challenge due to their diverge radio conditions, limited energy and computational capabilities. These were reasons for proposing fuzzy logic OF for dynamic adaptation of wireless network changing environment. Their results showed that fuzzy OF improved performance comparing to others in terms of throughput by $15 \%$ and $14 \%$ of (PDR) not mentioning energy consumption.

Another combined Fuzzy Logic OF was proposed by [11] Lamaazi and Benamar for overcoming the limitation of the standard OF which relies on single metric. The node and link metrics (i.e. Energy Consumption, Hop Count and Expected Transmission Count) were used in building the proposed OFEC. Effectiveness of newly proposed OF-EC was shown through simulations compared to MRHOF, ENTOT, and OFFUZZY. Major improvements were found in RPL performance in terms of PDR, convergence time, network lifetime, overhead, latency and energy consumption. It was found that OF-EC retained the efficiency of RPL totally independent from transmission rate \& network topology. Furthermore, OF-EC performed better than other proposals in terms of energy consumption among all nodes through network.

In [5] Sankar and Srinivasan, proposed an energy aware fuzzy logic RPL called (FLEA-RPL). It took several routing metrics into consideration; expected transmission count (ETX), residual energy (RER), and load, for choosing the best route. Fuzzy logic was applied over selected metrics to choose best route for efficient data transmission through network. Experiments were conducted using COOJA simulator for assessing proposed FLEA-RPL. Then set of comparisons with other similar protocols: MRHOF (ETX) based RPL (MRHOFRPL) and FL-RPL. Results showed 10-12\% improvement in: network lifetime and $2-5 \%$ in packet delivery ratio for FLEARPL.

\section{Choosing Metrics}

The metrics were chosen in order to get better performance measures. Using node metrics only cannot be able to accommodate the LLN constraints. As a result, combining link and node metrics is applied in this research paper. As previously stated, the proposed OFRRT-FUZZY considers the link and node metrics, namely, (RSSI), (RE) and (TH).

The major challenging requirements of RPL protocol are mobility support, reliable routing, energy-efficient routing and achieve higher throughput [24]. Usually sensors networks have several constraints; a critical one is that sensor nodes use batteries. Second, those sensors maybe deployed both unattended and in large numbers. That makes it difficult neither to change nor to recharge batteries in sensors. As a result, all processes, systems and communication protocols of sensor networks or sensors must take into consideration the minimizing power consumption. In addition, RSSI gives an indication of the power level that is received by the antenna. This means the higher RSSI level, the radio signal shall be stronger and thus, the destination shall be closer. (RSSI) can also be used as a measurement for wireless link quality [7]. Sriniv et al. [26] presents facts about RSSI in estimating link quality. RSSI is considered available during a packet reception with neither any impact on energy consumption, or additional hardware, nor throughput. In addition, this metric looks intuitive: stronger is the received signal, closer is the transmitter and weaker is the received signal, further is the transmitter. RSSI is employed in several standards for identifying the time on which the amount of radio energy in the channel is considered below a specific threshold at which point the node is clear to send.

Next section discusses the proposed OFRRT_FUZZY algorithm.

\section{The Proposed OFrRT-FuzZY AlgORITHM}

The OFRRT_FUZZY was developed based on fuzzy logic to enhance the performance results of the standard OFs (OF0 AND MRHOF) and choose the optimal path to reach the destination. Standard OFs usually depend on using only a single metric. the single metric approach doesn't meet all the application requirements [27][28]. Based on the node metric, OF0 is capable of providing a loosy quality of the link. While with MRHOF, the nodes shall provide a better link quality. However, it may be associated with a higher level of energy consumption. Due to these reasons, a combination of node and link metrics are proposed [23]. In particular, the proposed OFRRT-FUUZY uses fuzzy inference process(FIP), which is by definition "a process of mapping from a given input to an output, using the theory of fuzzy sets" [29]. Accordingly, the proposed method uses three input linguistic variables, namely, (RE), (TH) and (RSSI) to calculate a single output linguistic variable (Best path). The Mamdani-style FIP is applied to achieve this objective. It is one of the most commonly used fuzzy inference techniques. It includes four steps: crisp input fuzzification, rule evaluation, rule output aggregation, and defuzzification as shown in Fig. 2 the pseudo code of proposed Algorithm are discussed in Table I.

\section{A. Fuzzification}

In this step, crisp inputs, which are TH, RSSI and RE, are processed to determine the degree to which these inputs belong to each appropriate fuzzy set. Every linguistic variable has it is own Universe of Discourse (UOD), which determines its range of values. The value of a fuzzy set is provided based on the behavior of a linguistic variable. The fuzzy sets for the inputs and output are shown as follows:

$\mathrm{TH}=\{$ LOW, MID, HIGH $\}$

\section{RSSI $=\{$ CONNECTED,TRANSITIONING,DISCONNECTED \}}

\section{$\mathrm{RE}=\{$ SMALL, AVERAGE, HIGH $\}$}

Each linguistic variable has it is own UOD that clarifies its boundaries. The membership functions of the input linguistic variables (TH, RSSI and RE) are presented in Fig. 3, Fig. 4 and Fig. 5, respectively and the output linguistic variable (best path) are presented in Fig. 6. For computational simplicity, linguistic variables are frequently represented by triangles or trapezoids. A trapezoid is used in the proposed OFRRTFUZZY algorithm. The UOD for the TH input linguistic variable ranges from 0 to 1500 according to Maximum Transmission Unit (MTU). On the other side, the UOD for the 
RSSI input linguistic variable ranges from -50 to -100 , according to [30]. Last metric is RE the value is range between 0 to 250 as in [20]. The boundaries of the fuzzy sets and membership functions are chosen by domain experts [29].

\section{B. Fuzzy Rule Evaluation}

In this phase, the fuzzified inputs are applied to the rules based on knowledge, which is created by domain experts. In general, when the number of inputs increases in fuzzy logic, the complexity of rule application also increases. To solve this problem, multiple input single output is used [31]. To obtain a satisfactory rule base in fuzzy logic, rules should generally exhibit the following properties. First, rules should be complete, which indicates that rules must cover all system behavior. Second, rules should be consistent, which indicates that all rules must be logically valid. Each rule consists of two parts, the antecedent part of fuzzy rules, which is represented by "if" body rule, and consequent part (output). The antecedent part includes the input variables. Thus, the fuzzified inputs, together with their membership degrees, are applied to the first part to obtain the degree of membership in the consequent part. Some of rules are presented in Table II.

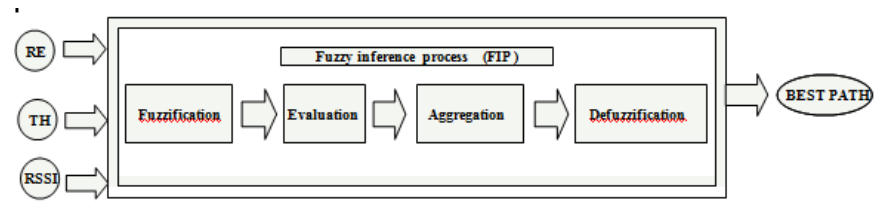

Fig. 2. Fuzzy Inference Process.

TABLE. I. PSEUdo CODE OF PROPOSED ALGORITHM

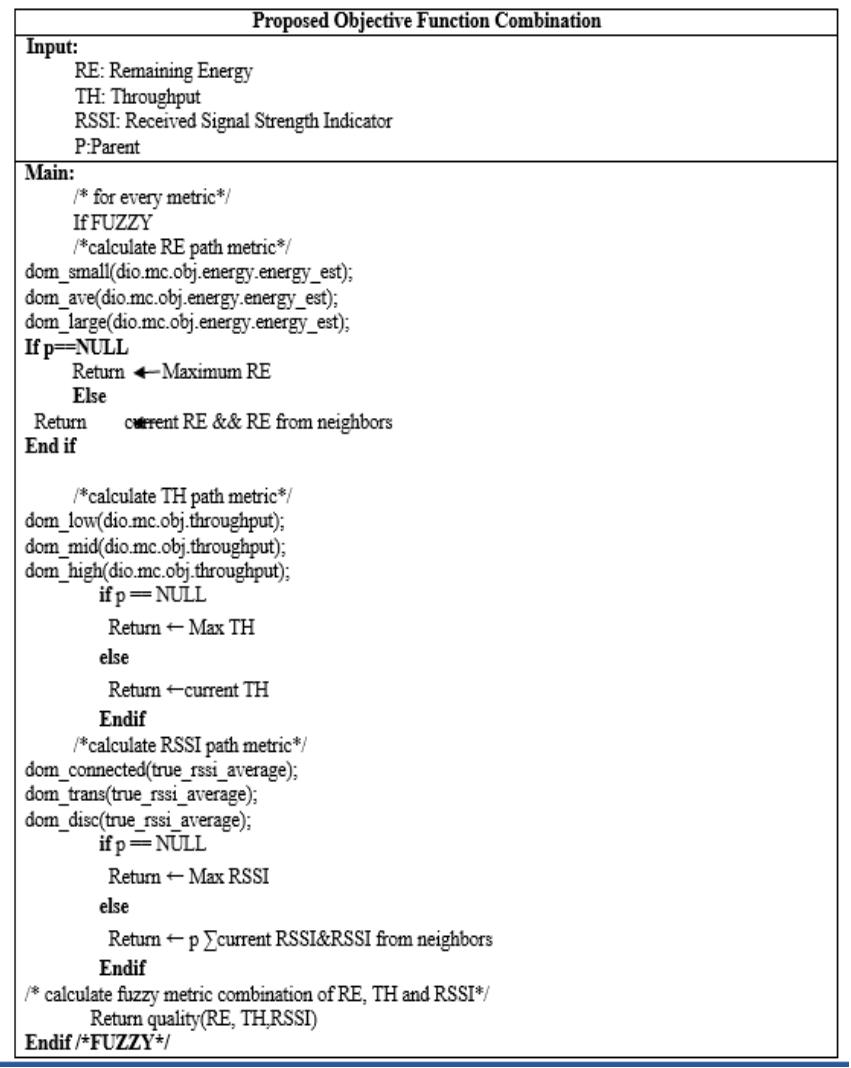

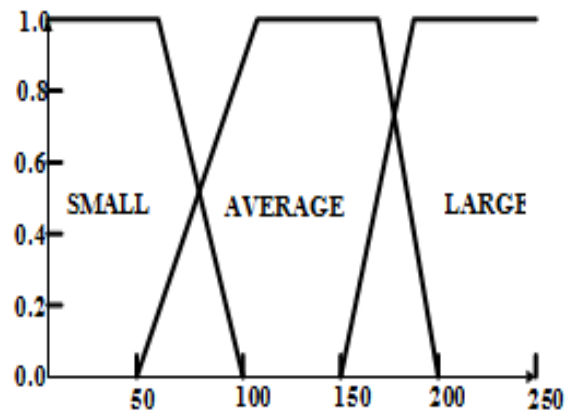

Fig. 3. Memberships Function of RE.

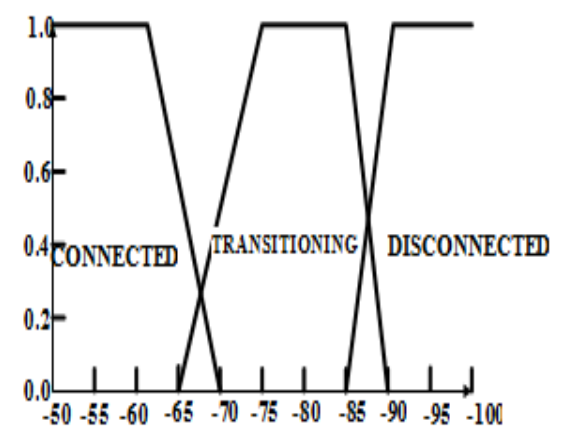

Fig. 4. Memberships Function of RSSL

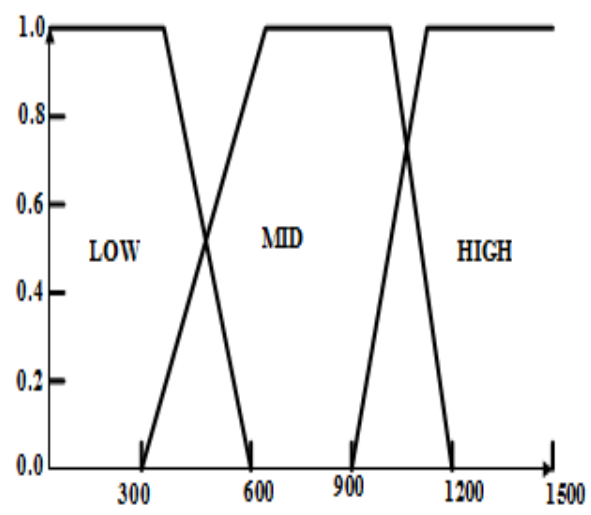

Fig. 5. Memberships Function of TH.

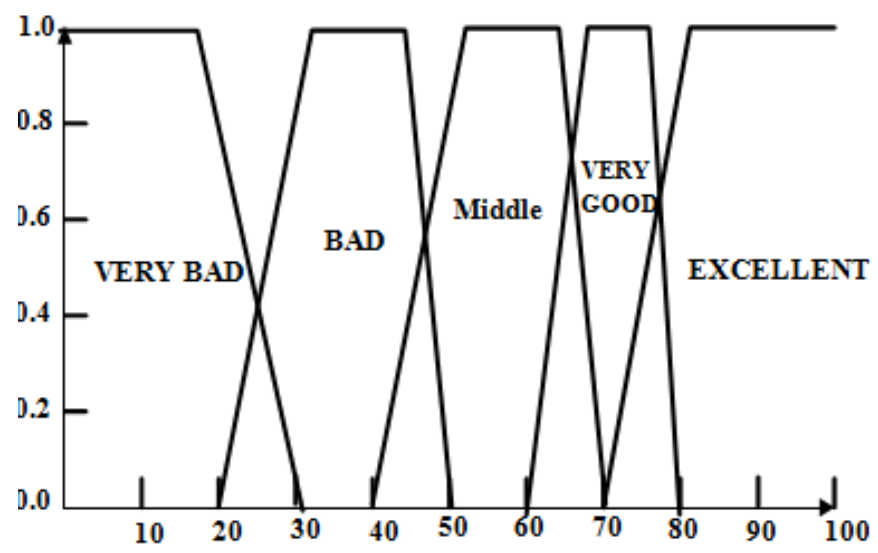

Fig. 6. Memberships Function of Best Path. 
TABLE. II. SOME OF FUZZY RULES FOR OFRRT-FUZZY

\begin{tabular}{|l|l|}
\hline Condition & Result \\
\hline IF RSSI is CONNECTED and this LOW and RE is SMALL & Bad \\
\hline IF RSSI is CONNECTED and this MID and RE is AVERAGE & Very Good \\
\hline IF RSSI is CONNECTED and this HIGH and RE is LARGE & Excellent \\
\hline IF RSSI is TRANSITIONIN and this LOW and RE is SMALL & Bad \\
\hline IF RSSI is TRANSITIONIN and this LOW and RE is AVERAGE & Middle \\
\hline IF RSSI is TRANSITIONIN and this LOW and RE is LARGE & Middle \\
\hline IF RSSI is DISCONNECTED and this LOW and RE is SMALL & Very bad \\
\hline $\begin{array}{l}\text { IF RSSI is DISCONNECTED and this LOW and RE is } \\
\text { AVERAGE }\end{array}$ & Bad \\
\hline IF RSSI is DISCONNECTED and this LOW and RE is LARGE & Bad \\
\hline
\end{tabular}

To obtain a good degree of output, the minimum operation will be adopted because the operation applied between the linguistic inputs is the "AND" operation for all the rules.

\section{Rule Output Aggregation}

Previously, a degree of membership is assigned to each consequent rule, and thus, combining all the outputs membership values from all the rules into a single fuzzy set is required. This process combines the inputs list of membership values with the single output fuzzy set for each output variable.

\section{Defuzzification}

The final step in FIP is to select a defuzzification method. The input for this step is the output from the previous step, which is a fuzzy set for every output linguistic variable, whereas the output from this step is a crisp value for each output linguistic variable. The defuzzfication method derives the crisp value to numerical value which is representing the fuzzy value of the linguistic output variable. In the OFRRTFUZZY proposed algorithm, the center of gravity (COG) is selected to find the numerical value of output. COG is the most popular approach because it finds the point at which a vertical line will divide the aggregate set into two masses that are equal [29]. COG is expressed in the following formula [29]:

$\mathrm{COG}=\frac{\sum_{\mathrm{a}}^{\mathrm{b}} \mu_{\mathrm{A}}(\mathrm{Si}) \times \mathrm{Si}}{\sum_{\mathrm{a}}^{\mathrm{b}} \mu_{\mathrm{A}}(\mathrm{Si})}$

where, $\mu \mathrm{A}(\mathrm{S})$ refers to the membership function of elements $\mathrm{Si}$ in sub-set $\mathrm{A}$ and $\mathrm{S}$ represent the degrees of membership.

Next section illustrate the performance evaluation of OFRRT_FUZZY

\section{Performance Evaluation}

\section{A. Simulation Setting}

A set of simulation experiments were designed for examining the performance level of the proposed OFRRTFUZZY in RPL protocol for IoT and compare it with the existing OFs such as OF0 and MRHOF. This simulation is carried out by COOJA simulation under Contiki OS. Contiki OS is an open source emulator that was designed for the IoT technology. Table III present the values for all parameters employed through the simulation experiments.
TABLE. III. COOJA SIMULATION PARAMETERS

\begin{tabular}{|ll|ll|}
\hline 20 & Network simulator & 21 & COOJA under Contiki OS (2.7) \\
\hline 22 & Objective function & 23 & OF0, MRHOF(ETX) and Proposed OF \\
\hline 24 & Number of nodes & 25 & $15,30,45$ \\
\hline 26 & Nodes type & 27 & Sky mote \\
\hline 28 & Topology & 29 & Grid \\
\hline 30 & $\begin{array}{l}\text { Radio medium } \\
\text { (Wireless Channel) }\end{array}$ & $31 \quad \begin{array}{l}\text { Unit Disk Graph Medium (UDGM) with } \\
\text { distance lose }\end{array}$ \\
\hline 32 & TX Ratio & 33 & $100 \%$ \\
\hline 34 & RX Ratio & 35 & $100 \%$ \\
\hline 36 & Transmission Range & 37 & 50 meter \\
\hline 38 & Interference Range & 39 & $100 \mathrm{~m}$ \\
\hline 40 & Simulation Times & $41 \quad$ Minutes \\
\hline
\end{tabular}

\section{B. Chosen Performance Metrics}

For evaluating the RPL performance level, the metrics must be chosen accurately to show the pros and cons of each RPL OFs. Therefore, the four metrics listed below were selected to evaluate The OFRRT_FUUZY, OF0 and MRHOF.

1) Average latency: It refers to the average time a transmitted packet consumes from sender node to sink node. The following equation can be used for calculating it:

Average latency $=\frac{\sum_{\mathrm{k}=1}^{\mathrm{n}} \operatorname{recv} \text { time }(\mathrm{k})-\text { send time }(\mathrm{k})}{\text { total packet received }}$

2) Packet delivery ratio $(P D R)$ : It refers to the ratio of nodes' number of received and sent packets. The following equation can be used for calculating it:

PDR $=\frac{\text { total received packets at the sink }}{\text { Total sent packets from senders }}$

3) Energy consumption $(\mathrm{mJ})$ : Energy anode requires to exchanges data through the network between nodes. The following equation can be used for calculating it.

Energy Consumption $=($ Transmit $* 19.5 \mathrm{~mA}+$ Listen $*$ $21.5 \mathrm{~mA}+$ CPU_time $* 1.8 \mathrm{~mA}+\mathrm{LPM} * 0.0545 \mathrm{~mA}) *$ $3 \mathrm{~V} /(32768)$

4) Control traffic overhead: Represents the total number of control messages DIO, DAO \&DIS used by ICMPv6, it is calculated based on the following equation.

Control Traffic Overhead $=\sum_{1}^{n} D I O+\sum_{1}^{n} D I S+\sum_{1}^{n} D A O(5)$

\section{Results and Discussion}

1) Average latency: The first evaluation metric for this study is to exam the impact of average latency for OFRRTFUZZY, OF0 and MRHOF with different size of network. Fig. 7 illustrates the measurement of average latency for each network size with different OF (OFRRY-FUZZY, OF0 and MRHOF). The result shows that the OFRRT-FUZZY has lower average latency as compare to $\mathrm{OF0}$ and MRHOF for each network size and MRHOF has higher average latency. In MRHOF case Select path from sender to sink based on ETX 
may get long time thus increase average latency. In case of OF0 select path from sender to sink based on number of hops will not decrease latency too much because some of nodes may get congested. In case of OFRRT-Fuzzy Select path from sender to sink based on combination of three matrices using fuzzy logic, less control traffic messages generated thus leads to less average latency.

2) Packet Delivery Ratio (PDR): Here the impact of PDR is exam for OFRRT-fuzzy, OF0 and MRHOF to observe the network reliability. The network size is varying. Fig. 8 Illustrates measure the PDR for each network size of each OF (OFRRT-Fuzzy, OF0 and MRHOF).

The result shows that OFRRT-Fuzzy has higher PDR as compare to OF0 and MRHOF for each network size and OF0 has low PDR. In OFRRT-Fuzzy allows for less packets loss due to use more than one metrics for selecting best path and this lead to higher PDR, MRHOF has more packet loss compare to OFRRT-Fuzzy due to continues calculation of best path. OF0 has the lower PDR, because it is produce more packets loss due to congestion that might occurs in the sender specially the senders that far from sink.

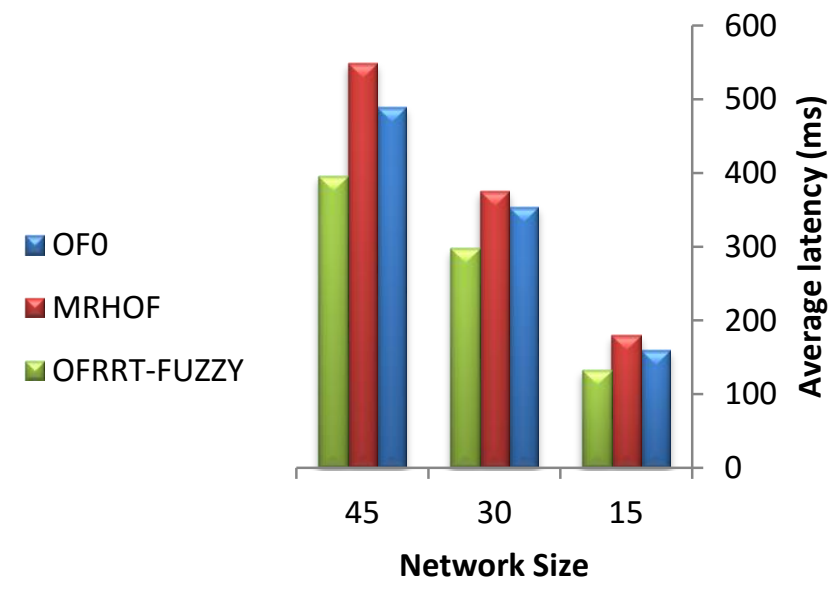

Fig. 7. Average Latency for OFO, MRHOF and OF-FUZZY.

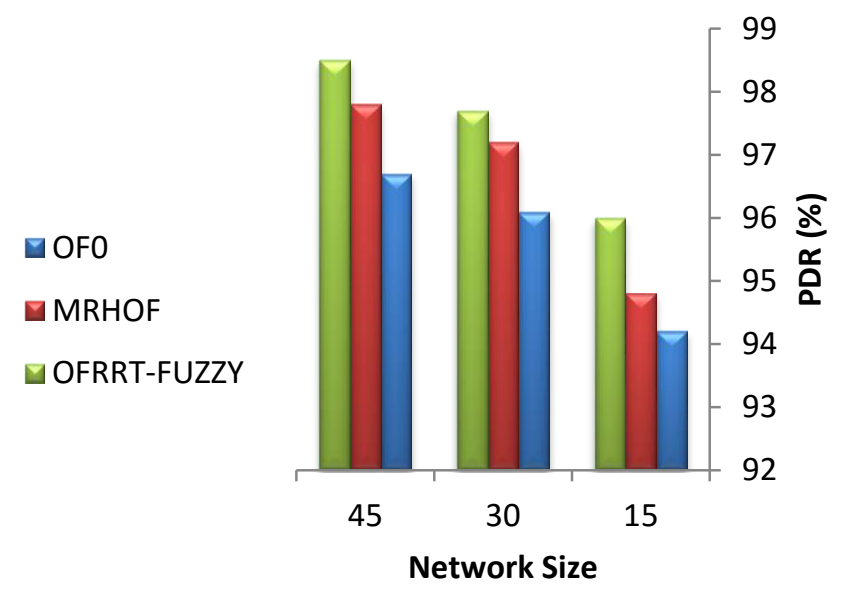

Fig. 8. Packet Delivery Ratio (PDR) For OF0, MRHOF and OF -FUZZY.
3) Energy Consumption $(\mathrm{mJ})$ : Here the impact of average power consumption is exam for OFRRT-FUZZY, OF0 and MRHOF. The network size is varying. The average power consumption calculating by considering the average power consumption used in each node for each network size of every OF (OFRRT-Fuzzy, OF0 and MRHOF).

Fig. 9 illustrates average power consumption for OFRRTFuzzy, OF0 and MRHOF of different network size, the result shows that the OFRRT-Fuzzy has lower average power consumption as compare to OF0 and MRHOF for each network size and MRHOF has higher average power consumption and with each network size and it is almost same as OF0. In OFRRT-Fuzzy allows less control message due using of combination metrics to select path from sender to skin therefore less CPU_ Power used relating to the node processing. HRHOF has higher average power consumption because the ETX calculation needed more process than hop and this lead to more average power consumption.

4) Control traffic overhead: In this section exam the impact of control traffic overhead for OFRRT-fuzzy, OF0 and MRHOF with different network size. The control traffic overhead is calculating by the sum of DIO, DIS and DAO for each network size of every OF (OFRRT-Fuzzy,OF0 and MRHOF).

Fig. 10 illustrates control traffic overhead for OFRRTFuzzy, OF0 and MRHOF of different network size, the result shows that OFRRT-Fuzzy has lower control traffic overhead as compare to $\mathrm{OF0}$ and MRHOF for each network size and MRHOF has higher control traffic overhead. RPL produce more control traffic messages during DODAG set up, Once DODAG is constructed, less ICMPv6 are produce. In OFRRTFuzzy Calculating combination of three metrics using fuzzy logic for select path from sender to skin needs less control messages as compared to MRHOF and OF0 because DODAG are constructed faster. MRHOF needs more control messages to complete DODAG constructed as compare to OF-FUZZY and OFO.

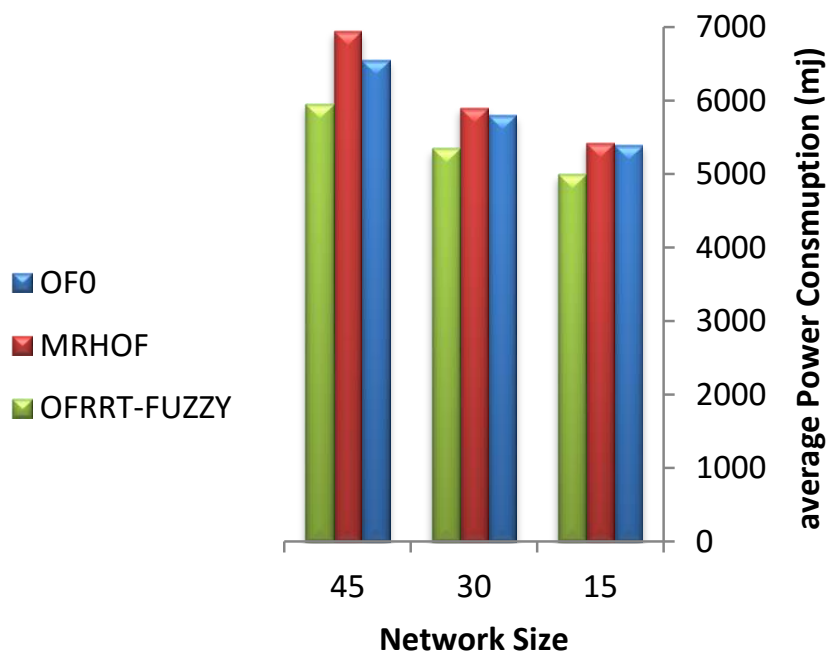

Fig. 9. Average Power Consumption for OF0, MRHOF and OF-FUZZY. 


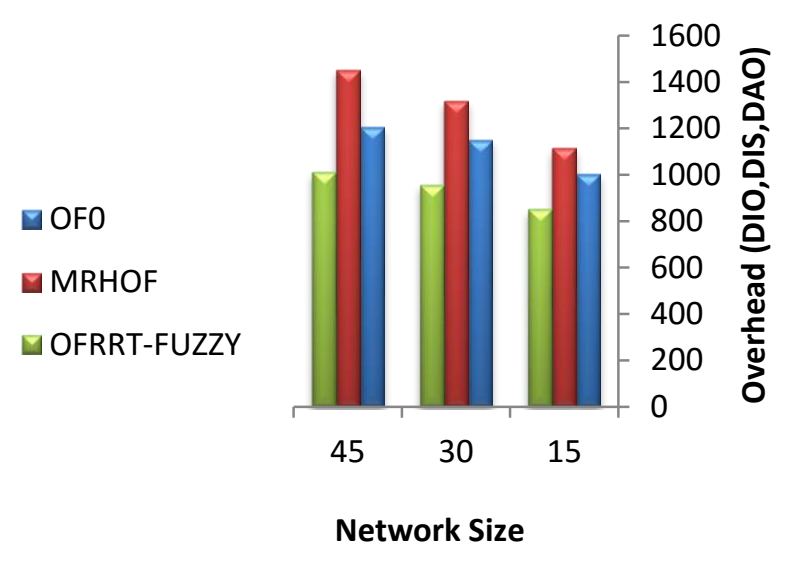

Fig. 10. Overhead for OF0, MRHOF and OF-FUZZY.

\section{CONCLUSION}

LLNs require certain type of protocols to work with. That is due to the fact of resource constraints. Mainly LLNs includes various types of devices which has different constraints as they are functioning. Standard routing protocol does not meet such needs. That is exactly why RPL was introduced. It is a distance vector protocol that finds the best path through OF. Many researchers are referred to in the related work section considered setting an OF with a single metric, that is either node or link. This paper proposed an OFRRT-FUZZY based on fuzzy logic which combines both link and nodes metrics namely; RE, RSSI and TH. The fuzzy logic method -which relies on a fuzzy membership which determines a set of rules for the combination-was adopted. Set of simulation experiments were conducted on Cooja and the results showed that OFRRT-fuzzy provide more satisfactory performance than OF0 and MHROF in terms of latency, PDR, overhead \& Power consumption.

\section{REFERENCES}

[1] S. Madakam, R. Ramaswamy, and S. Tripathi, "Internet of Things (IoT): A literature review," J. Comput. Commun., vol. 3, no. 05, p. 164, 2015.

[2] S. Agrawal and M. L. Das, "Internet of Things-A paradigm shift of future Internet applications," in 2011 Nirma University International Conference on Engineering, 2011, pp. 1-7.

[3] D. Airehrour, J. Gutierrez, and S. K. Ray, "Secure routing for internet of things: A survey," J. Netw. Comput. Appl., vol. 66, pp. 198-213, 2016.

[4] D. Airehrour, J. A. Gutierrez, and S. K. Ray, "SecTrust-RPL: A secure trust-aware RPL routing protocol for Internet of Things," Futur. Gener. Comput. Syst., vol. 93, pp. 860-876, 2019.

[5] S. Sankar and P. Srinivasan, "Fuzzy Logic Based Energy Aware Routing Protocol for Internet of Things," Int. J. Intell. Syst. Appl., vol. 10, no. 10, p. $11,2018$.

[6] M. D।'liaz, C. Mart'lin, and B. Rubio, "State-of-the-art, challenges, and open issues in the integration of Internet of things and cloud computing," J. Netw. Comput. Appl., vol. 67, pp. 99-117, 2016.

[7] S. Sankar and P. Srinivasan, "Composite Metric Based Energy Efficient Routing Protocol for Internet of Things," Int. J. Intell. Eng. Syst., vol. 10, no. 5, pp. 278-286, 2017.

[8] H. Lamaazi, N. Benamar, A. J. Jara, L. Ladid, and D. El Ouadghiri, "Challenges of the internet of things: IPv6 and network management," in 2014 Eighth International Conference on Innovative Mobile and Internet Services in Ubiquitous Computing, 2014, pp. 328-333.

[9] J. H. Kong, L.-M. Ang, and K. P. Seng, "A comprehensive survey of modern symmetric cryptographic solutions for resource constrained environments," J. Netw. Comput. Appl., vol. 49, pp. 15-50, 2015.
[10] H. Lamaazi, N. Benamar, M. I. Imaduddin, and A. J. Jara, "Performance assessment of the routing protocol for low power and lossy networks," in 2015 International Conference on Wireless Networks and Mobile Communications (WINCOM), 2015, pp. 1-8.

[11] H. Lamaazi and N. Benamar, "OF-EC: A novel energy consumption aware objective function for RPL based on fuzzy logic.," J. Netw. Comput. Appl., 2018.

[12] Z. Wang, L. Zhang, Z. Zheng, and J. Wang, "Energy balancing RPL protocol with multipath for wireless sensor networks," Peer-to-Peer Netw. Appl., vol. 11, no. 5, pp. 1085-1100, 2018.

[13] G. G. Lorente, B. Lemmens, M. Carlier, A. Braeken, and K. Steenhaut, "BMRF: Bidirectional multicast RPL forwarding," Ad Hoc Networks, vol. 54, pp. 69-84, 2017.

[14] P. Di Marco, G. Athanasiou, P.-V. Mekikis, and C. Fischione, "MACaware routing metrics for the internet of things," Comput. Commun., vol. 74, pp. 77-86, 2016.

[15] M. B. Yassein, S. Aljawarneh, and others, "A new elastic trickle timer algorithm for Internet of Things," J. Netw. Comput. Appl., vol. 89, pp. 38-47, 2017.

[16] M. Zhao, P. H. J. Chong, and H. C. B. Chan, "An energy-efficient and cluster-parent based RPL with power-level refinement for low-power and lossy networks," Comput. Commun., vol. 104, pp. 17-33, 2017.

[17] S. Tennina, O. Gaddour, A. Koubâa, F. Royo, M. Alves, and M. Abid, "Z-Monitor: A protocol analyzer for IEEE 802.15. 4-based low-power wireless networks," Comput. Networks, vol. 95, pp. 77-96, 2016.

[18] A. Oliveira and T. Vazão, "Low-power and lossy networks under mobility: A survey," Comput. networks, vol. 107, pp. 339-352, 2016.

[19] H. Fotouhi, D. Moreira, M. Alves, and P. M. Yomsi, "mRPL+: A mobility management framework in RPL/6LoWPAN," Comput. Commun., vol. 104, pp. 34-54, 2017.

[20] O. Gaddour, A. Koubâa, N. Baccour, and M. Abid, "OF-FL: QoS-aware fuzzy logic objective function for the RPL routing protocol," in 2014 12th International Symposium on Modeling and Optimization in Mobile, Ad Hoc, and Wireless Networks (WiOpt), 2014, pp. 365-372.

[21] P. O. Kamgueu, E. Nataf, T. Djotio, and O. Festor, "Fuzzy-based routing metrics combination for RPL," 2014.

[22] P.-O. Kamgueu, E. Nataf, and T. N. Djotio, "On design and deployment of fuzzy-based metric for routing in low-power and lossy networks," in 2015 IEEE 40th Local Computer Networks Conference Workshops (LCN Workshops), 2015, pp. 789-795.

[23] H. Lamaazi and N. Benamar, "RPL enhancement using a new objective function based on combined metrics," in 2017 13th International Wireless Communications and Mobile Computing Conference (IWCMC), 2017, pp. 1459-1464.

[24] E. Aljarrah, "Deployment of multi-fuzzy model based routing in RPL to support efficient IoT," Int. J. Commun. Networks Inf. Secur., vol. 9, no. 3, pp. 457-465, 2017.

[25] P. Fabian, A. Rachedi, C. Gueguen, and S. Lohier, "Fuzzy-Based Objective Function for Routing Protocol in the Internet of Things," in 2018 IEEE Global Communications Conference (GLOBECOM), 2018, pp. $1-6$.

[26] K. Srinivasan and P. Levis, "RSSI is under appreciated," in Proceedings of the third workshop on embedded networked sensors (EmNets), 2006, vol. 2006.

[27] P. O. Kamgueu, E. Nataf, T. D. Ndié, and O. Festor, "Energy-based routing metric for RPL," INRIA, 2013.

[28] J. P. Yunis and D. Dujovne, "Energy efficient routing performance evaluation for LLNs using combined metrics," in 2014 IEEE Biennial Congress of Argentina (ARGENCON), 2014, pp. 741-746.

[29] N. Michael, Artificial Intelligence A Guide to Intelligent Systems, vol. 321204662. 2005.

[30] I. H. Urama, H. Fotouhi, and M. M. Abdellatif, "Optimizing RPL objective function for mobile low-power wireless networks," in 2017 IEEE 41st Annual Computer Software and Applications Conference (COMPSAC), 2017, vol. 2, pp. 678-683.

[31] M.-S. Kim and S.-G. Kong, "Parallel-structure fuzzy system for time series prediction,” Int. J. Fuzzy Syst., vol. 3, no. 1, pp. 331-340, 2001. 